\title{
Muséologies
}

Les cahiers d'études supérieures

\section{Patrice Sauvé, Andrea Hauenschild et Lise Bertrand}

\section{Geneviève Angio-Morneau}

Volume 3, numéro 1, automne 2008

URI : https://id.erudit.org/iderudit/1033578ar

DOI : https://doi.org/10.7202/1033578ar

Aller au sommaire du numéro

Éditeur(s)

Association Québécoise de Promotion des Recherches Étudiantes en Muséologie (AQPREM)

ISSN

1718-5181 (imprimé) 1929-7815 (numérique)

Découvrir la revue

Citer ce document

Angio-Morneau, G. (2008). Patrice Sauvé, Andrea Hauenschild et Lise Bertrand. Muséologies, 3(1), 34-44. https://doi.org/10.7202/1033578ar d'utilisation que vous pouvez consulter en ligne.

https://apropos.erudit.org/fr/usagers/politique-dutilisation/ 


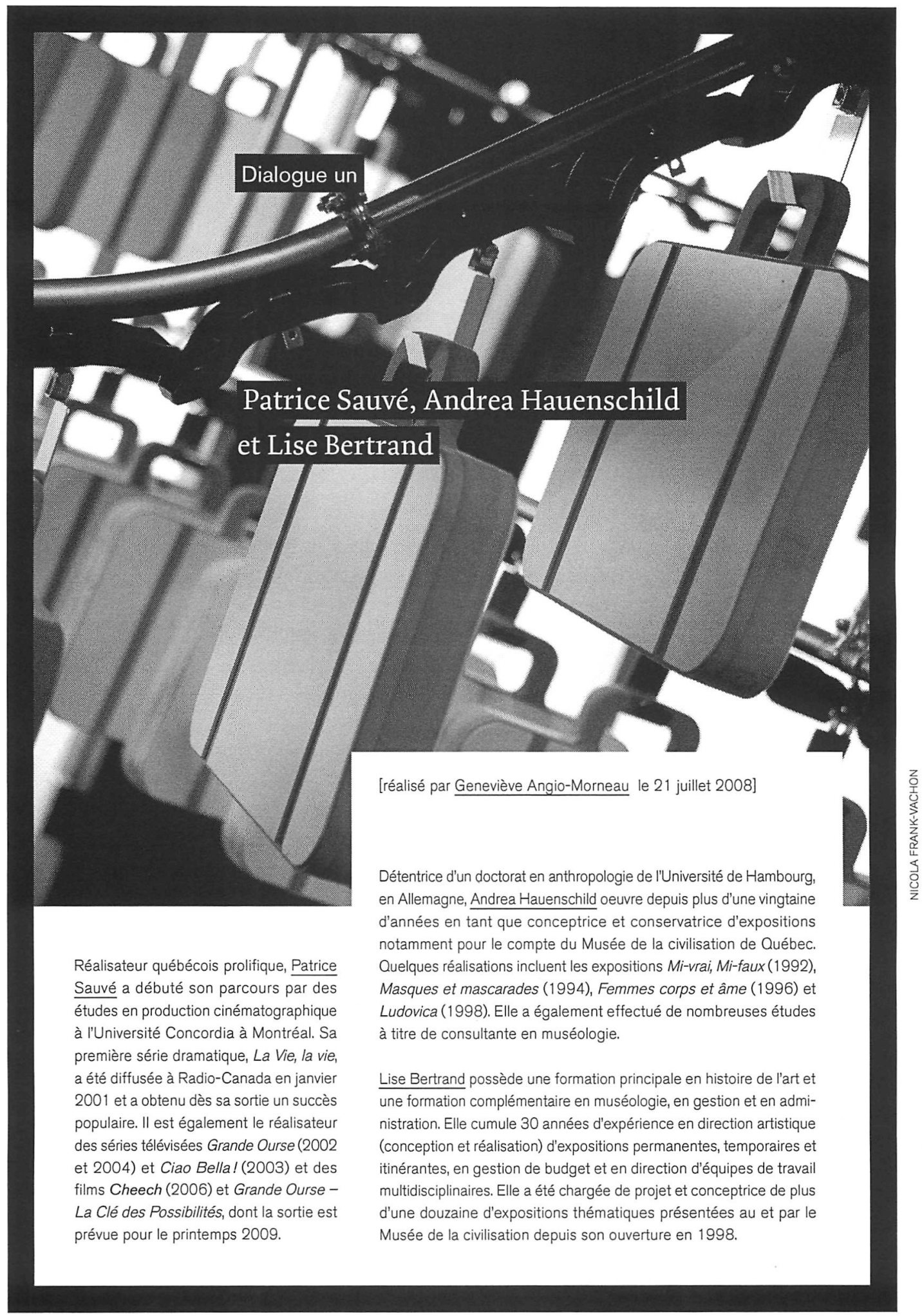


Conçu dans le cadre des célébrations du $400^{\mathrm{e}}$ de la Ville de Québec, l'exposition Passagers/Passengers célèbre la mouvance humaine à Québec. Habitant les espaces du nouveau bâtiment de Parc Canada situé sur le Bassin Louise- et nommé Espace $400^{\circ}$ pour l'été-, l'exposition rend hommage à la ville de Québec et à ses habitants à travers un parcours composé d'images, de témoignages, de paroles et de musique.

Geneviève AngIO-MoRneAu

Pouvez-vous nous décrire vos rôles respectifs dans la création de l'exposition Passagers/Passengers ?

\section{ANDREA HAUENCHILD}

Patrice était le concepteur principal, Lise était chargée de projet et moi conservatrice invitée. En travaillant avec Patrice et d'autres créateurs, nous avons assumé le rôle de commissaires.

\section{Patrice Sauvé}

Si je peux compléter, je crois que Lise et Andrea sont devenues petit à petit les "garantes" des intentions et de la cohérence du contenu. J'ai tendance à être assez prolifique au niveau des idées et beaucoup d'avenues ont été explorées.

GeneViève ANGIO-Morneau

D'où est venue l'idée d'aborder le thème de la mouvance humaine à travers le peuplement de la ville de Québec?

\section{Patrice Sauvé}

Avant même d'accepter de faire l'exposition, je suis allé voir le site où allait être construit le nouveau bâtiment de Parcs Canada avec Lise et les responsables de l'organisation du 4000 anniversaire de la ville de Québec. L'ancien bâtiment de Parcs Canada était encore là, et on m'a dit qu'il se trouvait surle lieu (avec la marina) dece qui était anciennement le site de débarquement des immigrants. C'est donc précisément sur ce sol que passaient les immigrants qui venaient s'installer à Québec ou qui allaient prendre le train vers d'autres destinations. Cet espace, ce site de l'ancien port, avait donc un sens, il était un lieu de passage, mais rien ne l'indiquait. C'est d'ailleurs une des raisons pour lesquelles nous avons souligné cette information dès l'entrée del'exposition.

L'idée de célébrer en ce le lieu la notion de mouvance humaine et de transition (et àl'origine nous discutions même de concepts tels arrivée/ départ, partir/rester) nous a semblé être un terrain fertile. Puis, en revenant de vacances, lorsquej'attendais mes bagages au carrousel à l'aéroport international Pierre-Elliott-Trudeau, j'ai eu le flash de l'aéroport et de tout le concept visuel et graphique del'exposition. M'est venue aussi l'idée de la valise. Je regardais passer les valises et je me disais qu'il y avait une vie dans chacune d'elles. C'est un classique, et bien des musées qui traitent d'immigration emploient ce symbole, mais la valise et l'aéroport sont tout de suite apparus comme un contexte pour parler de la mouvance humaine. Alors les deux se sont associés assez rapidement. 
J'avais aussi une autre idée, un plan B, mais la première a tout de suite beaucoup plu à Lise et à Andrea et à partir de ce moment nous avons commencé à explorer toutes les avenues possibles de cette idée. Nous nous sommes penchés sur la façon de celebrer Québec en utilisant des concepts clés : les arrivées, les départs, les naissances, les morts, les gens qui restent, le nombre de personnes, le nombre d'âmes, les nombres révelant toutes les facettes de cette immigration, la valeur des symboles, la valeur des quêtes individuelles... À cette étape, le plus grand problème était que nous avions beaucoup de faits historiques - et des chiffres -, mais peu d'écrits ou de témoignages provenant de gens ordinaires qui n'étaient pas des personnalités publiques et qui par conséquent ont laissépeu de traces écrites... Et c'est de là qu'est venue l'idée c'était même une nécessité et un désir - de faire parler des gens qui, aujourd'hui, avaient vécu une expérience de mouvance, afin de les mettre en relation avec l'histoire et de démontrer par ces témoignages que le passé et le présent se rejoignent en permanence.

\section{LISE BERTRAND}

Nous voulions faire appel à la mémoire pour constituer le corpus de l'exposition. J'avais vu l'exposition Tchernobyl, 20 ans après, où l'on avait interrogé des gens qui avaient survécu à l'accident. Notre réflexion s'inscrivait dans une démarche similaire de mémoire, mais par rapport à la mouvance. Nous nous sommes également posé la question à savoir ce qu'est une ville et nous sommes arrivés à la conclusion que c'est, entre autres, une accumulation de gens qui se succèdent. Nous avons donc fait calculer par un démographe le nombre de personnes qui étaient passées à Québec en 400 ans et le chiffre de 5 millions de personnes est ressorti. Le terme mouvance désigne aussi le phénomène de la migration humaine, universelle, actuelle et historique : dans ce mot on retrouve aussi la succession, la stratification des vies qui se sont superposées au fil des 400 ans de Québec.

\section{Patrice Sauvé}

Dansl'exposition, nous traitons de la mouvance intérieure, qui peut être naître ou mourir, quitter une famille, quitter un lieu, quitter un quartier tout simplement, et donc ce ne sont pas nécessairement des notions d'immigration, mais plutôt de déplacements intérieurs ou physiques que des individus vivent au cours de leur vie. Il faut dire par contre que Québec a été un port d'entrée important pourl'Amérique et par conséquent la ville de Québec est un lieu privilégié pour parler de mouvance physique. Je tenais à dire dans cette exposition que si nous sommes tous ici en ce moment, c'est qu'il y a quelqu'un parmi nos ancêtres qui a fait le chemin jusqu'à Québec, qui a tout quitté pour chercher un avenir meilleurici, et cela, nous devons le celebrer. Nous avons tous un bagage de mouvance en nous, mais nous n'y pensons pas. 

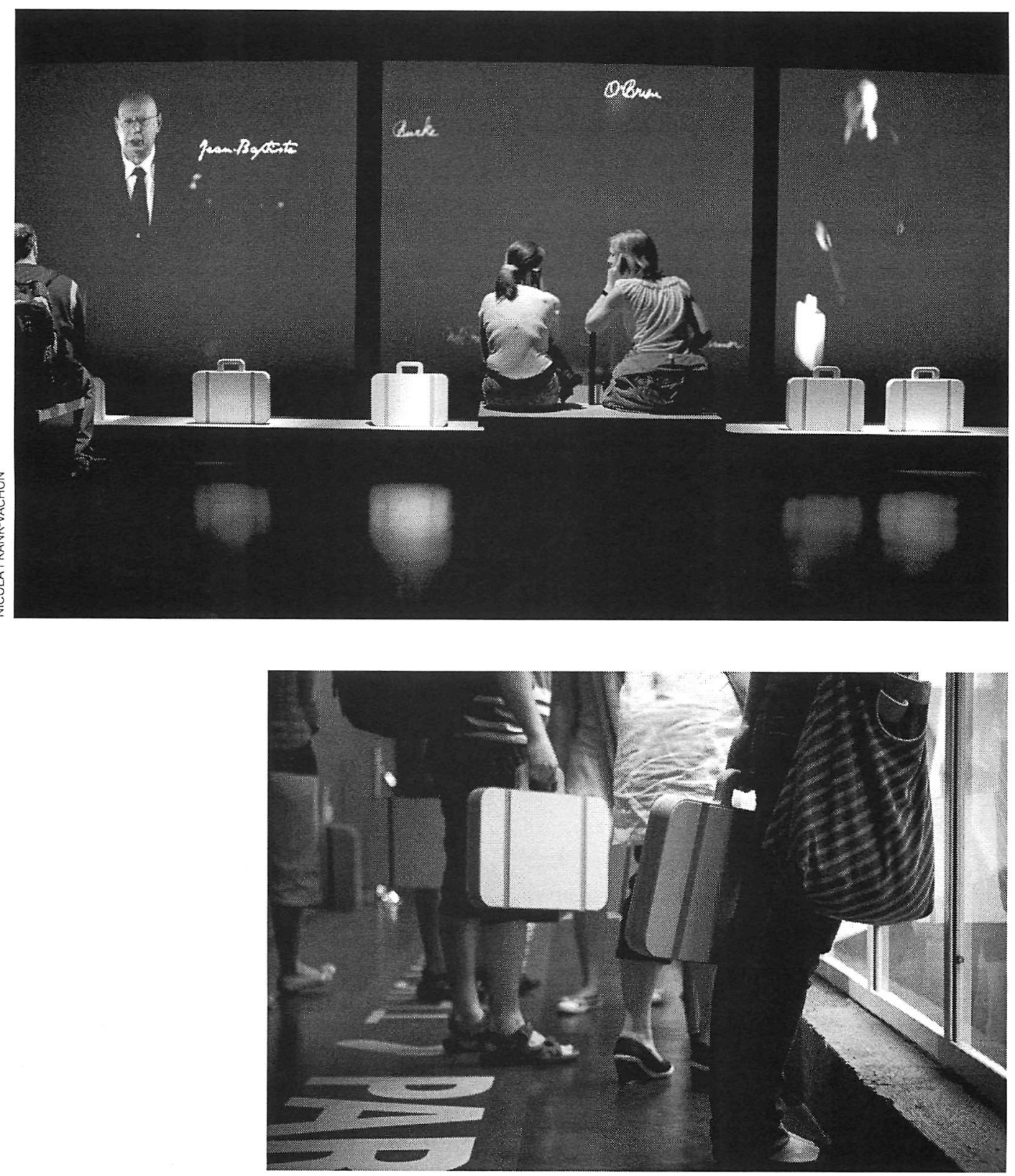
Geneviève ANGIO-MORNEAU

Comment avez-vous abordé la recherche en préparation pour cette exposition ?

\section{ANDRea HaUeNCHILD}

J'ai commencé par une vaste recherche exploratoire sur des expositions, des œuvres d'art et des installations, le conte, le thédtre, la photographie et d'autres moyens de communication. Il faut dire qu'en 1998 Lise et moi avions déjà réalisé ensemble l'exposition Ludovica surl'histoire de Québec, à l'occasion du $390^{\circ}$ anniversaire de la ville. Nous désirions renouveler notre approche et, puisque nous avions déjà collaboréà maintes reprises sur des projets, nous avons opté pour une grande recherche exploratoire.

LISE BERTRAND

Il faut dire qu'au départ nous ne savions pas exactement ce que nous allions faire puisqu'on nous avait donné carte blanche. Nous devions trouver l'angle pour parler d'une ville, pour la célebrer, pour commémorer son $400^{\circ}$. Alors en amont de toute recherche de contenu, nous avons fait une recherche sur la manière dont nous allions faire l'exposition. Nous sommes allées voir ce qui s'était fait ailleurs, dans des contextes semblables, au cours de la dernière année, que ce soit dans des expositions ou de grands événements. Nous avons aussi réuni notre comité scientifique, composé entre autres de Marc St-Hilaire, géographe, et de Marc Vallières, historien. Ce dernier nous a donné accès à une synthèse qu'il a assemblée de tout ce qui s'est fait en recherche sur l'histoire de la ville de Québec (qui n'est pas encore publiée). Marc St-Hilaire avait fait une étude surl'évolution de la population de Québec, et cela coïncidait parfaitement avec notre sujet. Nous sommes aussi allées à la rencontre d'archéologues et d'anthropologues, dont Serge Bouchard, anthropologue et animateur à Radio-Canada, pour comprendre comment ils percevaient un projet comme le nôtre, comment ils percevaient la ville de Québec, ce qui les frappait, ce que la ville avait de singulier pour eux.

\section{ANDREA HAUENCHILD}

À la suite de cette recherche sur la manière et $d u$ constat qu'il serait difficile - voire impossible - de faire une exposition d'objets, nous avons décidé de faire une exposition d'images, d'autant plus que le pavillon de l'Espace $400^{\circ}$ était un lieu non muséal qui n'offrait pas les conditions de conservation, de sécurité et d'entreposage habituelles. Nous voulions une exposition inspirée de l'histoire sociale qui mette en valeurles habitants de la ville de Québec, des gens que l'on ne trouve pas dans les livres d'histoire. Nous voulions que la population de Québec puisse se retrouver et se reconnaître dans l'exposition et nous avons choisi par conséquent de ne pas inclure les grands noms del'histoire. Sachant qu'il s'agirait d'une exposition d'images, nous avons fait appel à Patrice, réalisateur d'émissions de telévision novatrices, et nous avons constaté dès notre première rencontre que nous étions sur la même longueur 
d'ondes, que nous utilisions les mêmes référents, etc. Dès lors, nous avons organisé une réunion avec Marc Vallières et Marc Saint-Hilaire et nous avons ajouté d'autres spécialistes à l'équipe afin d'elaborer un contenu.

\section{Patrice Sauvé}

J'aimerais ajouter que la recherche ne s'est pas terminée à ce moment-là : nous avons dû poursuivre une fois le concept mis en place. Il a fallu trouver davantage d'information sur la mouvance, les arrivées, les départs, les naissances et les morts pour valider si nos idées tenaient la route. Il a aussi fallu voir comment nous allions définir, subdiviser et charger d'émotion les différentes vagues d'immigration. Ensuite, il fallait trouver des intervenants capables d'être représentatifs de ces vagues d'immigration et d'en parler. Lise Fournier, ethnologue, et Pascale Guéricolas, journaliste, ont parcouru la ville et ses différentes communautés pour identifier des personnes et faire des pré-entrevues. Puis il y a aussi eu le projet Farine Orpheline cherche Ailleurs Meilleur, où, à l'hiver 2007, on a installé un Photomaton dans différents lieux publics de la ville de Quebec pour recueillir dans cette boîte à images près de 200 témoignages de monsieur et madame Tout-le-Monde, qui ont accepté de livrer leur expérience de mouvance en disant d'où ils venaient, qui étaient leurs ancêtres et à quel moment ils étaient arrivés à Québec.
ANDREa HaUenchild

En tout, nous avons recueilli environ 400 témoignages desquels nous en avons retenu une soixantaine pour figurer dans l'exposition.

Geneviève Angio-Morneau Passagers/Passengers est une exposition sans objets muséaux. Quels étaient les enjeux?

ANDREA HAUENCHILD

Il est vrai que Passagers/Passengers est une exposition sans objets dans le sens habituel du terme, mais je dirais plutôt qu'ils sont tout simplement différents. D'abord, le témoignage devient objet d'exposition. Il y a ensuite l'image vidéo, qui est une création, et finalement il y a la scénographie de l'image, c'est-à-dire la mise en espace de l'image dans l'exposition, qui se distingue de la manière dont on est habitué de voir les images dans une salle de cinéma. Dans notre cas, le visiteur-spectateur circule dans l'espace, dans un parcours composé d'images.

\section{PATrice SAuvé}

Il faut dire qu'au départ nous voulions intégrer des objets à l'exposition. Nous avions eu l'idée d'intégrer des jouets qui représentaient la mouvance : trains, autobus, autos, charrettes etc. Nous voulions aussi créer une caravane composée de minuscules objets, comme des petits personnages. Nous avons par ailleurs imaginé une maquette incroyable avec des petits personnages qui côtoieraient les visiteurs et des silhouettes en déplacement... Mais nous n'avons pas réussi 
à les intégrer à l'exposition; en faisant le deuil de ces objets nous avons eu l'idée de demander à un artiste de créer une œuvre qui celébrerait la mouvance de façon tangible. Finalement, il y a eu un concours...

ANDREa HaUenchild

Pour célebrer la mouvance humaine de Québec, nous pensions àl'assemblage d'objets de mémoire et s'est alors présentée la possibilité d'organiser un concours en collaboration avec le Conseil des arts et des lettres du Québec (CALQ), ouvert à tous les artistes. Au terme du processus de sélection, nous avons choisi André Dubois de Quebec. Celui-ci a créé une immense sculpture qui est suspendue dans l'atrium du pavillon, au centre du parcours de l'exposition. Cette sculpture représente une référence matérielle aux témoignages, à la mémoire qui est relatée dans les images vidéo...

\section{LISE BERTRAND}

[...] et aussi à la géographie de la ville, à son histoire ainsi qu'à des objets personnels. C'est comme une synthèse de l'histoire de cette ville que nous habitons et une matérialisation de la mouvance.

Geneviève Angio-Morneau

Comment avez-vous composé avec le lieu de l'exposition?

\section{ANDREA HAUENCHILD}

C'est intéressant parce que le lieu de l'exposition représentait un défi majeur. Lors du premier concept, le pavillon n'était pas encore en rénovation ou en construction et l'ensemble du processus de conception et de scénarisation s'est déroulé alors qu'on ne pouvait pas y pénétrer. Nous n'avions jamais vu l'espace et c'est seulement après le "concept final » qu'il nous été possible de le visiter pour une première fois. Il y a eu surprises et ajustements qui, de toute façon, font partie du processus. L'espace a aussi représenté un défi sur le plan du contenu parce que l'exposition s'articule sur quatre étages et est, par conséquent, spatialement fragmentée. Il était donc important de créer un parcours qui retiendrait l'attention du visiteur du début à la fin, $y$ compris dans les espaces de transition.

\section{PAtrice SAUvé}

C'est vrai que faire "àl'aveugle " n'était pas évident. Nous avons louédes gymnases et avons fait des marques au sol pour essayer de comprendre l'espace. Pour moi c'était vraiment important parce que, au lieu de créer une histoire en deux dimensions comme j'ai l'habitude de faire,j'ai dû comprendre cette tridimensionnalité très particulière au langage d'exposition, qui se fait dans des volumes qui doivent se répondre les uns les autres. Les espaces permettaient de créer un vrai chemin dramatique : bien qu'il n'y ait pas de trame dramatique comme dans une fiction traditionnelle, je voulais qu'elle puisse se percevoir au niveau du scénario. Je voulais que le visiteur devienne passager dès le debut de l'exposition et qu'ensuite il ait un chemin à prendre. Le passager 
commençait par monter dans un ascenseur, un sas, et entrait progressivement dans une réalité altérée qui lui permettait de faire une transition entre la réalité et la transposition. Ensuite, il y avait une salle de mouvance, une salle de celebration pour les arrivées et finalement une dernière salle, difficile à décrire avec les mots, qui en était une de célébration plus poétique de l'arrivée, une espèce de réincarnation pour le visiteur de sa propre présence à Québec.

Geneviève ANGIO-MORNEAU

On sent vraiment que Passagers/Passengers est un projet où la spécificité du lieu a été déterminante dans le développement du concept et du scénario.

Patrice Sauvé

Oui, et c'est aussi parce que l'espace existant $d u$ bâtiment et que ses matériaux, plus particulièrement sur la façade extérieure, donnaient une impression d'un aéroport moderne. Faire ce genre de transposition devenait aisé.

Geneviève Angio-Morneau

Pouvez-vous nous parler du processus de filmer l'ensemble des individus dont les témoignages vidéo se retrouvent dans l'exposition?

\section{Patrice Sauvé}

Nous avons sélectionné, parmi les entrevues réalisées, celles qui se démarquaient par leur histoire et nous en avons assemblé ainsi une variété qui était représentative (quant à leur origine, leur sexe et leur age) de la population de la ville de Quebec. Une fois les personnes choisies, il a fallu instaurer une infrastructure de tournage qui convienne au budget. Il faut comprendre que nous devions standardiser le lieu de tournage (même chaise, même éclairage, même gestes posés au-delà de l'entrevue) afin d'obtenir 50 entrevues similaires. Ensuite, un protocole a été mis en place pour bien prendre en charge ceux qui avaient accepté de faire l'entrevue. Il était important que ces participants comprennent bien ce que nous attendions d'eux et que tout cela sefasse dans un contexte rempli de bienveillance et de tendresse afin qu'ils se sentent comme dans un écrin. C'était un gros défi parce qu'il n'y a rien de plus désespérant qu'une entrevue où quelqu'un est figé, ce qui arrive fréquemment avec des gens qui n'ont pas l'habitude de passer devant la caméra. Nous devions donc nous assurer que le contact puisse se faire entre la personne interviewée et le visiteur del'exposition qui les écoute et qui n'a que 30 secondes pour saisir leur âme. Je dois dire que je suis particulièrement fier $d u$ résultat.Je n'ai pas fait ces entrevues, j'ai confié la tâche à la réalisatrice Françoise Lavoie-Pilote de la maison de production numérique 6ixdegrés; quandj'ai vu les premiers rushes sortir et que j'ai vu les gens, je suis resté profondément ému du fait qu'ils semblaient jaser avec quelqu'un qui leur était familier :j'ai compris alors qu'on m'avait donné ce que je souhaitais.

Andrea Hauenchild

J'aimerais ajouter que Lise et moi avons assisté au tournage de ces témoignages qui s'est déroulé de façon assez intense pendant cinq jours. Il 
s'agit sans aucun doute d'un des moments les plus émouvants du projet de voir toutes ces personnes défiler devant la caméra, devant nous, nous expliquant chacune son expérience de mouvance, et puis livrer son bagage personnel, témoignant de façon très généreuse, sincère et authentique. Je veux aussi souligner le fait que je crois que Patrice a réussi, avec la collaboration de Françoise Lavoie-Pilote, à réinventer l'esthétique de l'audiovisuel muséal ; lorsqu'on examine de plus près la facture de ces très courts enregistrements, on peut percevoir un autre objet muséal, qui est à la fois une installation vidéo et une composition d'éléments de cinéma.

Geneviève Angio-Morneau

L'exposition met davantage l'accent sur les immigrants arrivés à Québec que sur les émigrants ou sur les gens de passage. Quelle est la raison derrière ce choix ?

LISE BERTRAND

Grâce à Marc Vallières, nous nous sommes aperçus que c'était très facile de retracer les arrivants car il existe beaucoup de documents et d'archives. Il devient par contre plus difficile de documenter les départs parce que les gens n'avertissent personne lorsqu'ils partent. C'est ce manque de ressources sur les départs qui a fait que nous avons demandé aux interviewés de nous parler de leur lieu d'origine avant leur départ pour Québec. Nous avons pu de cette façon contourner le problème et les faire parler d'un départ malgré tout, même si cela a été fait de façon sommaire.

\section{Geneviève Angio-MorneaU}

Patrice, décrivez-nous l'expérience de travailler avec un média autre que la télévision et le cinéma.

\section{Patrice Sauvé}

Je trouve que le média de l'exposition est beaucoup moins directif.Je ne peux pas être aussi despote que je peux l'être au cinéma! C'est vrai qu'à la télé il y a les pauses commerciales et le pouvoir de la zapette; au cinéma, les gens peuvent quitter la salle après que s'éteignent les lumières, même si c'est difficile. Je dirais que ce qui a été particulièrement intéressant en exposition c'est que l'expérience est moins linéaire. En vidéo, il y a une linéarité dans la selection des plans où l'on travaille avec plusieurs couches vidéo ou audio et puis, de toute évidence, à la fin on n'en monte qu'une seule. Dans le cas de Passagers/ Passengers, j'avais le droit de doubler, de tripler, de quadrupler, et même plus, les niveaux vidéo. C'était extrêmement intéressant parce que le temps devient infini, c'est-à-dire que le visiteur peut décider de passer 30 secondes, cinq minutes ou 50 minutes dans une même salle et de faire l'expérience de chaque niveau de la vidéo. Il peut avoir une première impression et ne garder que celle-là ou il peut décider de rentrer progressivement dans des niveaux d'intimité de contenus plus approfondis.

Ce qui est commun à l'exposition, au cinéma et à la télévision, c'est la charge émotive. Je construis tout ce que je fais en conservant une charge émotive que j'essaie de traduire ensuite 
par les moyens que j'ai à ma disposition et ce fut la même chose pour Passagers/Passengers. J'avais vraiment un sentiment instinctif permanent pourl'ensemble de l'exposition et pour chacune des salles quej'ai essayé de préserver tout au long du scénario. Bien sûr, plusieurs chemins ont été explorés au cours de l'année que nous avons passée à la conception, mais nous avons toujours gardé ce que nous nommons " un petit feu " pour chacune des salles.

J'ajouterais que cela été un privilège de pouvoir articuler cette expérience en trois dimensions, dans un autre cadre temporel et avec une complexitéde signaux qui lui est propre. Deux niveaux narratifs se complétaient dans Passagers/Passengers : le premier niveau, celui de l'émotion, était plus impressionniste ou poétique et il était composé des témoignages et de la façon dont j'ai essayé de les envelopper. Le second niveau était composé de la signalétique de l'aéroport qui a été détournée aux fins del'exposition. Oui c'était del'information, mais elle devenait de plus en plus ludique au fur et à mesure que l'on progressait dans l'exposition. On a joué avec les codes de la signalétique et ceux qui ont voyagébeaucoup dansles aéroports pourront sourire en voyant le traitement qu'on a fait, que ce soit dans les vidéos d'une hôtesse de l'air que l'on retrouvait à plusieurs endroits sur le parcours des visiteurs ou bien dans les écrits et les pictogrammes des panneaux graphiques. Quant àl'hôtesse, non seulement elle guidait les visiteurs physiquement, en leur donnant des directives, mais elle les incitait à réfléchir en posant des questions liées à la salle suivante. Bien sûr, il fallait trouver l'équilibre afin que cela devienne le contrepoint à l'émotion et que l'ensemble soit fait avec un brin d'humour.

Geneviève Angio-Morneau

Lise et Andrea, comment ce projet s'est-il démarqué de vos projets antérieurs?

LISE BERTRAND

Pour Andrea et moi, il s'agissait d'une exposition très "expérimentale ". Nous étions dans un contexte inhabituel, dans un lieu hors $d u$ musée et nous travaillions avec un créateur qui venait du milieu du cinéma. Nous travaillions un peu sans filet, nous n'avions pas l'équipe du Musée de la civilisation à notre disposition et nous avons dû constituer notre propre équipe. De plus, nous avions choisi une voie non traditionnelle puisque nous avions décidé de jouer entre l'installation vidéo/art contemporain et l'exposition thématique. Nous avons voulu conjuguer ces deux concepts en maintenant une bonne communication avec le visiteur, en n'allant pas trop loin dans l'installation, qui est souvent hermétique et difficile à saisir, et en allant au bout de la synthèse et de la recherche pourdiffuser aux visiteurs une expérience comme cela se fait plus communément lors de la visite d'une exposition thématique. 
Geneviève AngIo-Morneau

Pour conclure, pouvez-vous élaborer un peu sur ce qu'a représenté pour vous Passagers/Passengers?

\section{Patrice SaUvé}

J'ai énormément appris. Je n'avais jamais fait d'exposition auparavant et j'avais des préoccupations (des angoisses !) en ce qui avait trait aux espaces, à la façon de les meubler et de les habiter. Il y a aussi la méthodologie de travail qui est différente, qui est un peu plus posée. Il y a ce sentiment d'urgence, presque permanent en audiovisuel traditionnel, que ce soit en cinéma ou en télévision. C'était très bien d'avoir Andrea et Lise à mes cotés car cela permettait un certain recul qui est toujours difficile à avoir dans un processus créatif. Entre les étapes, on avait le temps de confronter ce qu'on proposait etj'ai trouvé cela positif. Et puis, il y a aussi ce que je n'avais pas fait depuis l'école de cinéma, écrire un scénario, alors que dans mon travail de réalisateur je prends habituellement le scénario de quelqu'un d'autre pour le traduire en audiovisuel. Bien que mes œuvres audiovisuelles réussissent à être personnelles, je crois sincèrement que Passagers/Passengers est vraiment l'une des choses les plus personnelles que j'aie offertes au public jusqu'à présent.
LISE BERTRAND

Pournous qui sommes en muséologie, l'expérience a été un stage intensif en matière de technologie audiovisuelle. Nous n'avions jamais vu de plateau de tournage ni travaillé avec un réalisateur. Nous avons approuvé des rushes, des montages, des bandes sonores et des effets spéciaux. Nous avons maintenant une autre vision de ce qu'est une production audiovisuelle.

\section{ANDREa HaUeNCHILD}

Lise et moi avons aussi beaucoup appris surce qui constituait le sens dramatique, c'est-à-dire la construction d'une courbe dramatique, la progression d'une narration et aussi sur ce que pouvait être le traitement et l'utilisation de l'image vidéo et des témoignages dans le cadre d'une exposition thématique. Je veux aussi revenir surle thème de la mouvance humaine, parce queje pense que nous nous sommes tous identifiés (Patrice, Lise et moi) et retrouvés dans ce thème, dans cette exposition, en tant que personnes en mouvement, venues d'ailleurs nous-mêmes où par nos ancêtres. Nous avons souvent échangé surnos histoires personnelles qui ressemblent en fin de compte aux histoires de nos témoins dans l'exposition. En ce sens, l'expérience humaine a été extrêmement enrichissante. 